\title{
EL ÉTER RELATIVISTA: UN CAMBIO CONCEPTUAL INCONCLUSO
}

\author{
Alejandro Cassini \\ Universidad de Buenos Aires/CONICET \\ alepafrac@yahoo.com.ar \\ MARCELO LEONARDo LEVINAS \\ Universidad de Buenos Aires/CONICET \\ Instituto de Astronomía y Física del Espacio (IAFE) \\ leo@levinas.com.ar
}

RESUMEN: La posición inicial de Einstein con respecto al éter electromagnético y su posterior eliminación del escenario de la relatividad especial resultaron profundamente alteradas por la relatividad general. En este artículo analizamos los diferentes argumentos de Einstein contra el éter electromagnético. Luego estudiamos las razones que lo llevaron a reintroducir el éter gravitatorio en la relatividad general. Después de comparar las propiedades y funciones del antiguo éter con las del nuevo éter relativista, concluimos que se ha producido un cambio conceptual y que el término "éter" ha experimentado un cambio de significado. No obstante, mostramos que el nuevo éter retuvo algunas de las propiedades y funciones características del viejo éter electromagnético.

PALABRAS CLAVE: relatividad especial, éter gravitatorio, espacio-tiempo, relatividad general, cambio de significado

SUMMARY: Einstein's initial attitude towards electromagnetic ether in special relativity was deeply disrupted by general relativity. In this paper we analyze Einstein's different arguments against electromagnetic ether in the frame of special relativity. We then study his reasons for postulating a gravitatory ether in general relativity. After comparing the properties and functions of the traditional electromagnetic ether with those of the new relativistic ether, we conclude that a conceptual change has occurred, because the term "ether" has undergone a change of meaning. However, we also show that the new ether still retains many properties and functions characteristic of the old electromagnetic ether.

KEY WORDS: special relativity, gravitatory ether, space-time, general relativity, change of meaning

\section{Introducción}

A la mayoría de los físicos actuales el éter de la electrodinámica clásica, como cualquier otro tipo de éter, les parece un fantasma del pasado que Einstein conjuró para siempre en 1905. Hoy casi todos admiten que los diferentes campos físicos cuya existencia postulan nuestras teorías son entidades autosubsistentes que no necesitan un soporte material, ni, mucho menos, uno de carácter mecánico. No 
obstante, cuando Einstein formuló la relatividad especial (RE), la comunidad de los físicos creía, de manera casi unánime, que el éter era imprescindible como sustrato del campo electromagnético. En 1905, Einstein declaró prescindible al éter, pero dejó sin explicar cómo podía propagarse la luz en el espacio vacío sin algún soporte material. Durante más de una década pensó que el éter debía desterrarse para siempre del dominio de la física, mientras que los físicos se negaron a abandonarlo, por lo menos hasta después de 1930. A partir de 1916, después de completar la formulación de la teoría de la relatividad general (RG), Einstein reivindicó la existencia del éter. Sostuvo que la RG implicaba la existencia de un éter gravitatorio y revisó parcialmente su posición respecto de la $\mathrm{RE}$, afirmando que ésta no era incompatible con algunas características del antiguo éter electromagnético.

El éter que Einstein reivindicaba no era, por cierto, el éter material de la electrodinámica clásica. Era, por el contrario, un éter desprovisto de toda propiedad mecánica, del cual no podía decirse siquiera que estuviera en estado de reposo. Su argumento principal para resucitar al éter era que la RG atribuye al espacio-tiempo propiedades físicas bien determinadas, como los potenciales gravitatorios, incluso en regiones desprovistas de toda materia ponderable. Por consiguiente, este espacio-tiempo no es en absoluto un vacío en un sentido tradicional, sino una entidad física que adquiere el carácter de un éter inmaterial. Así, según Einstein, el éter, el espacio-tiempo y el campo gravitatorio debían concebirse como una y la misma cosa. Pocos físicos aceptaron la reintroducción de este éter relativista. Señalaron, con razón, que no se trataba de un éter material como el de la electrodinámica clásica, sino de un tipo diferente de entidad, que sólo de manera equívoca o arbitraria podía llamarse "éter". Es indudable que la propuesta de Einstein implicaba un cambio de significado en el concepto mismo de éter, pero este hecho por sí solo no volvía inaceptable el uso del término. Casi todos los conceptos fundamentales de la física, por ejemplo, los de fuerza, masa y energía, cambiaron de significado a lo largo de la historia y no dejamos por ello de utilizarlos en nuestras teorías actuales. Por otra parte, el concepto mismo de éter cambió varias veces de significado desde el siglo XVII, y se encontraba en pleno proceso de cambio en los años que precedieron a la formulación de la RE. ¿Estaba entonces justificada la decisión de Einstein de llamar "éter" al espacio-tiempo relativista, o se trataba, simplemente, de una mera cuestión de palabras que podía resolverse de manera convencional? 
En este trabajo nos proponemos analizar la introducción del éter relativista en su contexto histórico. Mostraremos, en primer lugar, cómo el concepto de éter se había vuelto inestable hacia fines del siglo XIX y se encontraba experimentando un proceso de cambio de significado, ya evidente en la electrodinámica de Lorentz. En segundo lugar, sostendremos que si la posición de Einstein respecto del éter se enfoca como la culminación de este proceso, el concepto de un éter relativista representa en algunos aspectos un cambio radical de significado respecto del concepto tradicional de éter electromagnético. Finalmente, intentaremos probar que, a la vez, el éter relativista retiene varias de las características y funciones del éter clásico, por lo que resulta razonable llamarlo con este nombre.

El trabajo está organizado de la siguiente manera. En la sección 2 argumentamos que, en la electrodinámica clásica, el éter desempeñaba tres funciones diferentes, por lo que una teoría que pretendiera desterrarlo debería mostrar que es posible prescindir de todas esas funciones, algo que Einstein evidentemente no hizo en su trabajo fundacional de 1905. En la sección 3 analizamos los diferentes argumentos que posteriormente formuló Einstein para justificar la eliminación del éter en la RE. Concluimos allí que él ofrece argumentos para prescindir del éter en cuanto referencial absoluto y sustrato del campo electromagnético, pero no ofrece ningún argumento a favor de la eliminación de la acción a distancia. En la sección 4 exponemos las razones de Einstein para introducir la idea de que la RG implica la existencia de un éter gravitatorio y para retractarse de su afirmación de que la RE era incompatible con la existencia del éter electromagnético. En la sección 5 exponemos la tesis de Einstein sobre el éter relativista en el contexto de los debates sobre la naturaleza y las propiedades del éter electromagnético, y mostramos de qué manera todo ello se relaciona con el proceso de cambio de significado del concepto de éter. En la sección 6 nos preguntamos si la introducción del éter gravitatorio implica una concepción sustancialista del espaciotiempo. En las conclusiones señalamos que el cambio de significado producido por la relatividad general constituye un verdadero cambio conceptual y mostramos por qué el éter relativista de Einstein debe retener ciertas propiedades características del éter material de la electrodinámica clásica.

\section{Las funciones del éter en la electrodinámica clásica}

Desde los comienzos del pensamiento científico y filosófico, el éter se concibió como una sustancia material imponderable que llenaba el 
espacio aparentemente vacío, en particular, los espacios interestelares. La postulación de esta materia sutil obedeció, según los diferentes autores, a las más diversas razones tanto físicas como metafísicas. Como señaló Maxwell, de los diferentes tipos de éteres imaginados por científicos y filósofos, hacia mediados del siglo XIX "el único éter que ha sobrevivido es el que fue inventado por Huygens para explicar la propagación de la luz" (Maxwell 1875, p. 764). El éter luminífero apareció como una necesidad conceptual para la teoría ondulatoria de la luz. Esta teoría, en todos sus representantes principales (Huygens, Euler, Young y Fresnel), se desarrolló sobre la base de una analogía entre la luz y el sonido: así como el sonido es una vibración del aire, la luz debe ser una ondulación en algún medio material. El éter luminífero resultaba, entonces, un presupuesto necesario cuando la luz se concebía como una especie de onda mecánica.

Las tres funciones principales del éter en la electrodinámica clásica eran: a) evitar la acción a distancia, b) servir de sustento para la propagación de los campos electromagnéticos, y c) proporcionar un referencial privilegiado en el cual se hallaban expresadas las ecuaciones de Maxwell.

Para que el éter pudiera llevar a cabo la función de evitar la acción a distancia debía llenar todo el espacio, incluso el espacio entre las partículas materiales que componían los cuerpos y, posiblemente, también el interior de las propias partículas. Como consecuencia de ello, debía penetrar todos los sólidos. Se sigue de allí que debía ser una sustancia continua, pues, si tuviera una estructura atómica, la acción a distancia tendría que producirse entre los átomos mismos del éter. ${ }^{1}$

Para desempeñar la función de sustento del campo electromagnético, el éter debía tener propiedades mecánicas inusuales. Originalmente, la analogía de la luz con el sonido llevó a postular la hipótesis de que las ondas luminosas eran ondas de presión longitudinales en un éter luminífero. Sin embargo, desde comienzos del siglo XIX, la explicación de fenómenos como la difracción y la polarización condujo a Fresnel a postular que la luz se propagaba bajo la forma de ondas transversales. Por ello no podía suponerse que el éter era un fluido, sino que debía ser un sólido sumamente elástico, ya que sólo los sólidos de esta clase podían transmitir ondas transversales a una velocidad muy alta como la de la luz. Además, tenía que ser extrema-

\footnotetext{
${ }^{1}$ Así, todos los modelos mecánicos del éter en los que se postula una estructura atómica o molecular de esta sustancia, como los del propio Maxwell, permiten evitar la acción a distancia a escala macroscópica, pero no a escala microscópica.
} 
damente rígido para poder transmitir las ondas a esa velocidad. Por otra parte, tenía que ser incompresible, porque no debía transmitir ondas longitudinales, como la materia ordinaria. Finalmente, el éter luminífero no debía oponer ninguna resistencia al movimiento de la materia, que parece desplazarse a través de él sin pérdida alguna de energía debida la fricción. Ningún tipo de sólido conocido tenía simultáneamente todas estas propiedades, por lo que pronto se advirtió que el éter tenía que ser una especie muy rara de materia sutil dotada de propiedades mecánicas muy especiales, aparentemente incompatibles entre sí. Ningún intento de ofrecer un modelo mecánico del éter, de los muchos que se hicieron a lo largo del siglo XIX, resultó exitoso o siquiera verosímil. ${ }^{2}$

Además del éter luminífero, se postularon otros tipos de éter, como el eléctrico y el magnético, concebidos como mediadores de las respectivas interacciones que, aparentemente, también actuaban a distancia. A partir de la síntesis producida por la electrodinámica de Maxwell, donde la óptica, la electricidad y el magnetismo se unificaron en una sola teoría, los tres éteres quedaron reducidos a uno solo: el éter electromagnético, soporte de las ondas electromagnéticas, de las cuales la luz no era más que una clase particular. Después de Maxwell, los campos eléctrico y magnético se concibieron como simples estados del éter electromagnético.

Por último, la función del éter de proporcionar un referencial privilegiado era una consecuencia de la electrodinámica de Maxwell, en la que la velocidad de propagación de las ondas electromagnéticas aparecía como una constante física. ${ }^{3}$ Pero, como se sabía desde Newton, las velocidades de los cuerpos no eran invariantes, sino relativas a un referencial determinado. La interpretación más natural de la constante $c$, manteniendo el marco de la mecánica newtoniana, suponía que dicha velocidad era relativa al éter electromagnético y que, por consiguiente, las ecuaciones de Maxwell eran válidas en un referencial que se encontrara en reposo respecto de dicho éter.

En la electrodinámica de Maxwell, la velocidad de la luz sólo podía referirse entonces a un referencial en reposo respecto del éter. Las transformaciones de Galileo, incorporadas a la mecánica newtoniana,

\footnotetext{
${ }^{2}$ Sobre los diferentes modelos del éter electromagnético, véanse Schaffner 1972, y Cantor y Hodge 1981. La obra enciclopédica de Whittaker (1951 y 1953), aunque envejecida en muchos aspectos, todavía es indispensable como visión de conjunto.

${ }^{3} \mathrm{La}$ velocidad de las ondas electromagnéticas en el vacío (o, más bien, en el éter) es $c=\left(1 / \varepsilon_{0} \mu_{0}\right)^{1 / 2}$ (donde $\varepsilon_{0}$ es la permitividad eléctrica del vacío y $\mu_{0}$ es la permeabilidad magnética del vacío).
} 
implicaban, en efecto, que si la velocidad de la luz tenía un determinado valor respecto de dicho referencial, dicho valor debía ser diferente en cualquier otro referencial inercial que se encontrara en movimiento uniforme respecto del éter. Por consiguiente, las ecuaciones de Maxwell no eran invariantes bajo las transformaciones de Galileo, y, asumiendo que la Tierra se movía respecto del éter, dichas ecuaciones no debían ser válidas en un referencial terrestre. De esta manera, la formulación de una electrodinámica aplicable a los cuerpos en movimiento se convirtió en uno de los problemas fundamentales para los sucesores de Maxwell, y, como se sabe, fue el problema que condujo a la RE de Einstein.

$\mathrm{Si}$ el éter electromagnético era una sustancia material, entonces debía tener un estado dinámico bien determinado. El problema de la movilidad del éter, y de su arrastre parcial o total por los cuerpos que se mueven a través de él, permaneció sin resolverse a lo largo de todo el siglo XIX. No obstante, era un problema legítimo para la física en tanto el éter se concibiera como una especie de materia, diferente del espacio mismo aunque ocupándolo completamente. En el marco de la física prerelativista era perfectamente significativo preguntarse por el movimiento absoluto, tanto de los cuerpos ponderables como del propio éter a través del cual dichos cuerpos se movían. Como es bien conocido, hubo innumerables experimentos ópticos y electromagnéticos que intentaron determinar el movimiento de la Tierra respecto del éter, de los cuales el de Michelson y Morley de 1887 fue posiblemente el más influyente. ${ }^{4}$ Como suele ocurrir, el resultado negativo de todos estos experimentos se prestó a muchas interpretaciones diferentes, de las cuales el éter totalmente arrastrado por los cuerpos de Hertz (1890) y la contracción de los cuerpos en la dirección del movimiento en un éter en reposo absoluto de Lorentz (1895) representan dos posiciones extremas.

Retrospectivamente, resulta evidente que la hipótesis de la existencia del éter electromagnético provocaba una serie de problemas, tanto de carácter teórico como experimental, que ninguna teoría entonces vigente había logrado resolver de manera satisfactoria. No resulta extraño, entonces, que la existencia misma del éter pudiera cuestionarse.

\footnotetext{
${ }^{4}$ Los experimentos que se proponían detectar el viento de éter comenzaron mucho antes de la formulación de la RE y continuaron hasta mucho después de ella. Para una descripción detallada véase Swenson 1972.
} 


\section{La eliminación del éter en la relatividad especial}

En el estado en el que se encontraba la física inmediatamente antes de la formulación de la RE había varias razones para dudar de la existencia del éter. En primer lugar, por los reiterados resultados negativos de todos los experimentos destinados a detectar el movimiento relativo de la Tierra respecto del éter, que continuaron mucho después del de Michelson y Morley. Es indudable que Einstein conocía estos resultados experimentales, al menos indirectamente a través de la obra de Lorentz, y los mencionó genéricamente al comienzo de su artículo de 1905 y más explícitamente en la segunda formulación de la RE en 1907.5 En segundo lugar, por las dificultades ya señaladas para elaborar un modelo mecánico del éter que diera cuenta de sus particulares propiedades físicas. La proliferación de modelos incompatibles, casi siempre meramente especulativos e incluso con un indisimulable carácter ad hoc, seguramente fomentó las dudas acerca de la realidad del éter. Incluso los defensores de la hipótesis del éter hacía tiempo que habían reconocido la ausencia de una teoría satisfactoria acerca de la composición de esta sustancia y las escasas probabilidades de obtenerla en el futuro. ${ }^{6}$ En tercer lugar, por el auge del energetismo, que, aunque minoritario en la comunidad de los físicos, minaba una de las razones fundamentales por la cual se consideraba necesario postular la existencia del éter. Según los energetistas, la energía, en cualquiera de sus formas, era una sustancia que existía con independencia de cualquier soporte material, por lo que el éter no resultaba necesario para la propagación de las ondas electromagnéticas. De hecho, la energía se postuló como la única sustancia de la naturaleza, de lo cual resultó un programa que se proponía desplazar a la mecánica como fundamento de la física. Si bien este programa se hallaba en decadencia ya a comienzos del siglo XX, había dejado sus huellas en Alemania y, sobre todo por medio de la obra de Ostwald, había influido en la formación inicial

${ }^{5}$ El papel que desempeñó el experimento de Michelson y Morley (1887) en la génesis de la RE es una cuestión muy discutida. En 1905, Einstein no mencionó el experimento (1905b, p. 891); en 1907, en cambio, lo hizo de manera explícita (1907, p. 411). Se sabe con certeza que, antes de 1905, Einstein había leído el libro de Lorentz (1895), donde se discute este experimento. Sobre la manera en que Einstein lo reinterpretó, véase Cassini y Levinas 2005.

${ }^{6}$ Tanto Lorentz (1902) como Michelson (1903, p. 163) lo admiten con cierta resignación. Poco antes, Larmor había señalado que dado que no hay manera alguna de experimentar sobre las propiedades del éter libre, en la práctica es como si el éter no tuviera estructura (Larmor 1900, p. 188). 
de Einstein. ${ }^{7}$ En cuarto y último lugar, por la difusión de la epistemología positivista que propugnaba la eliminación de las entidades inobservables del dominio de las teorías físicas, ya fueran el espacio y tiempo absolutos, los átomos o las fuerzas atractivas y repulsivas entre los cuerpos. Parece evidente que el éter, que se había resistido a todo intento de observación, podía caer fácilmente bajo el programa eliminativista de los positivistas. Es un hecho bien conocido que, a través de la obra de Mach, la filosofía del positivismo influyó de manera decisiva en la formación intelectual de Einstein y en la formulación de las teorías especial y general de la relatividad. El propio Einstein reconoció en muchas ocasiones el valor de las ideas de Mach (quien paradójicamente no rechazó la hipótesis del éter) como guía para la elaboración de las dos teorías de la relatividad. ${ }^{8}$

Es un lugar común afirmar que la formulación de la teoría de la RE significó el final del éter electromagnético o, al menos, el inicio de un proceso rápido de decadencia para las teorías del éter. En efecto, al comienzo mismo de su artículo fundacional de 1905, después de enunciar los dos postulados de la teoría, el principio de relatividad y el de constancia de la velocidad de la luz, Einstein dice lo siguiente:

Estos dos postulados son suficientes para llegar a una electrodinámica de los cuerpos en movimiento simple y consistente sobre la base de la teoría de Maxwell para los cuerpos en reposo. La introducción de un "éter luminífero" se mostrará superflua, en tanto que, de acuerdo con el concepto que se desarrollará aquí, no se introducirá ningún "espacio en reposo absoluto" dotado de propiedades especiales, ni se asignará un vector velocidad a un punto del espacio vacío en el cual ocurren los procesos electromagnéticos. (Einstein 1905b, p. 892)

Éste es el único pasaje que Einstein dedica al éter en su formulación original de la RE. Se lo considera usualmente como el acta

${ }^{7}$ Sabemos que en su juventud Einstein leyó la obra de Ostwald (1893) y la citó en su primer trabajo científico publicado (Einstein 1901). Ostwald había afirmado que la existencia del éter era innecesaria en cuanto sustrato de la energía electromagnética ya que "la energía radiante debe reconocerse como autosubsistente en el espacio" (Ostwald 1893, vol. 2, p. 1016).

${ }^{8}$ La influencia exacta de Mach sobre el pensamiento de Einstein ha sido muy debatida, pero es innegable (véase Holton 1968 y Sánchez Ron 1983, pp. 207-217). Einstein admite el papel de Mach en su formación intelectual en su autobiografía, escrita en 1946 (Einstein 1949) y en una carta a Michele Besso del 6 de enero de 1948, aunque para esa época rechazaba sus ideas epistemológicas. Mucho antes, en 1916, había llegado a decir que Mach estuvo muy cerca de formular una teoría de la relatividad general (Einstein 1916c, p. 103). 
de defunción del éter; pero esta evaluación, según creemos, es puramente retrospectiva. Podemos advertir inmediatamente que Einstein no afirma de manera explícita que la existencia del éter sea incompatible con la RE, ni mucho menos que esta teoría implica que el éter no existe. Sólo dice que, en el marco de la RE, el éter resulta "superfluo", esto es, innecesario. Desde nuestro punto de vista, esto significa que el éter no desempeña ninguna función en la RE y que, por consiguiente, es posible prescindir de la hipótesis que postula su existencia. Hemos dicho, sin embargo, que en la electrodinámica de Maxwell y Lorentz el éter desempeñaba tres funciones. En consecuencia, para probar que, en el contexto de la RE, la hipótesis del éter es verdaderamente superflua es necesario mostrar que no desempeña ninguna de esas tres funciones. Esto, evidentemente, no lo hace Einstein en el artículo de 1905, dado que el pasaje que acabamos de citar se dirige solamente contra una de las funciones del éter: la de proporcionar un referencial privilegiado, en reposo absoluto, respecto del cual son válidas las leyes de Maxwell, Einstein no explica, en 1905, la razón por la cual la RE no requiere un referencial en reposo absoluto aunque es evidente que se sigue del principio de relatividad mismo. Sólo en 1909 Einstein formuló de manera explícita este argumento con las siguientes palabras:

El principio de relatividad establece, entonces, que todas las leyes de la naturaleza que se refieren a un sistema de coordenadas $K^{\prime}$ en movimiento uniforme respecto del éter sean iguales a las correspondientes leyes referidas a un sistema de coordenadas $K$ en reposo respecto del éter. Pero si esto es así, entonces, tenemos la misma razón para imaginar al éter en reposo respecto de $K^{\prime}$ como en reposo respecto de $K$. Por lo tanto, es completamente antinatural seleccionar uno de los dos sistemas de coordenadas $K^{\prime}$ introduciendo un éter en reposo respecto de él. De eso se sigue que sólo se puede obtener una teoría satisfactoria si se renuncia a la hipótesis del éter. (Einstein 1909, p. 819)

Este argumento, que luego Einstein repitió en numerosas ocasiones, ${ }^{9}$ implica que, en el contexto de la RE, las ecuaciones de Maxwell no necesitan un referencial en reposo absoluto, como el referencial galileano que había supuesto Lorentz. Sin embargo, el argumento no prueba que sea posible prescindir de las otras dos funciones que el éter desempeñaba en la electrodinámica clásica. La RE no hace ninguna afirmación explícita respecto de la naturaleza de la luz y,

${ }^{9}$ Por ejemplo, en Einstein 1920a y 1920b. 
en principio, es compatible con una teoría corpuscular de la luz, en la cual, obviamente, la existencia del éter sería innecesaria. Sin embargo, en una concepción ondulatoria de la luz, como la de Maxwell y Lorentz, la supresión del éter como referencial inercial privilegiado no implica, sin más, que el éter sea prescindible como sustrato de las ondas electromagnéticas.

La RE tampoco implica por sí misma la eliminación de la acción a distancia, como ocurre en las teorías de campos. Ello es así porque no es necesariamente una teoría de campos, aunque su origen se halle ligado a problemas de la teoría del campo electromagnético de Maxwell y Lorentz. La RE simplemente impone restricciones cinemáticas para cualquier otra teoría, ya sea mecánica, electromagnética o de otra clase. Así, por ejemplo, en cualquier teoría relativista las leyes deben ser invariantes bajo las transformaciones de Lorentz y ninguna interacción podrá propagarse con una velocidad superior a la de la luz en el vacío. No obstante, acerca del mecanismo de transmisión de las interacciones, la RE no afirma nada específico, en particular, no afirma que las interacciones se propaguen a través de campos continuos. En principio, entonces, la RE debería ser compatible con una electrodinámica de acción a distancia entre partículas y, en general, con cualquier teoría de acción a distancia en la que se cumpla la cinemática relativista.

La teoría del cuanto de luz de 1905a, reformulada en el marco de la RE, le hubiese permitido a Einstein prescindir simultáneamente de las tres funciones del éter. En efecto, los cuantos de luz, concebidos como una suerte de partículas de carácter semiclásico, no necesitan el éter como sustrato material, puesto que pueden propagarse en el espacio vacío. Los cuerpos, entonces, podrían interactuar electromagnéticamente mediante la emisión y la absorción de cuantos de luz que se mueven siempre con una velocidad invariante $c$ (por lo que deberían tener una masa en reposo nula). Con ello se eliminaría, además, la necesidad de postular acciones a distancia, ya que toda interacción electromagnética resultaría mediada por los cuantos de luz (que es lo que sucede con los fotones en las teorías cuánticas de campos). Resulta intrigante, pues, que Einstein no haya apelado a su teoría del cuanto de luz, formulada sólo tres meses antes de la RE, cuando argumentó en contra de la necesidad de postular la existencia del éter luminífero. ${ }^{10}$

\footnotetext{
${ }^{10}$ Es de destacar el hecho de que, en 1905a, Einstein mencionó el éter cuando se refirió a la interacción entre la radiación y la materia. Un análisis detallado de las diferencias conceptuales entre los artículos 1905a y 1905b de Einstein puede encontrarse en Cassini y Levinas 2007 y 2008.
} 
En la segunda exposición de la RE, escrita a fines de 1907, Einstein ofreció otro argumento contra la necesidad de suponer la existencia del éter, que formuló de esta manera:

la concepción de un éter luminífero como vehículo de las fuerzas eléctricas y magnéticas no encaja en la teoría aquí descrita, pues los campos electromagnéticos aparecen aquí no como estados de alguna materia, sino más bien como cosas autosubsistentes similares a la materia ponderable y que tienen en común con ella la propiedad de la inercia. (Einstein 1907, p. 413)

En ese momento, Einstein pareció pensar que la eliminación del éter como referencial absoluto implicaba también su eliminación como sustrato del campo electromagnético. Los siguientes pasajes, escritos en 1909, confirman esta conjetura, porque siguen inmediatamente a la exposición del argumento contra el éter como referencial absoluto que ya antes hemos comentado in extenso: "Entonces, los campos electromagnéticos que constituyen la luz ya no aparecen más como estados de un medio hipotético, sino como entidades autosubsistentes emitidas por las fuentes de luz, precisamente como en la teoría newtoniana de la emisión de la luz" (Einstein 1909, p. 819).

Einstein concluyó, entonces, que: "La teoría de la relatividad ha cambiado también nuestras concepciones de la naturaleza de la luz en cuanto no concibe la luz como una secuencia de estados de un medio hipotético, sino como algo autosubsistente, tal como la materia" (Einstein 1909, p. 820).

Desde nuestro punto de vista, este último argumento es independiente de cualquier consideración relativista. La idea de que las líneas de fuerza eléctricas y magnéticas puedan existir en el espacio sin necesidad de un soporte material ya la había formulado Faraday cuando, en 1846, conjeturó que la radiación podría originarse en la vibración lateral de estas líneas de fuerza y extrajo la conclusión de que ello permitiría eliminar el éter (Faraday 1846, p. 451). En principio, entonces, es posible sustancializar el campo electromagnético en el contexto de la propia teoría de Maxwell, sin apelar a ninguna consideración relativista. Es verdad que la eliminación del éter en cuanto referencial inercial privilegiado puede motivar también su eliminación como sustrato de las ondas electromagnéticas, pero es evidente que una cosa no se sigue de la otra.

En 1920, Einstein formuló de la siguiente manera el argumento que apoya la eliminación del éter como sustrato material de las ondas electromagnéticas: 
Los campos electromagnéticos no son estados de un medio, sino que son realidades autosubsistentes que no son reducibles a otra cosa ni están ligados a ningún portador, exactamente como los átomos de la materia ponderable. Esta concepción se propone también porque, según la teoría de Lorentz, la radiación electromagnética lleva consigo impulso y energía, como la materia ponderable, y porque la materia y la radiación, según la teoría de la relatividad especial, son ambas solamente formas especiales de energía distribuida, por lo que la materia ponderable pierde su posición privilegiada y aparece sólo como una forma especial de energía. (Einstein 1920b, p. 9)

El argumento de Lorentz era un argumento mecánico, mientras que el de Einstein (que apela a la equivalencia entre masa y energía) es relativista; se trata de un argumento lógicamente independiente de todos los que Einstein había formulado anteriormente. La equivalencia entre masa y energía la había derivado en 1905c, donde había determinado que una fuente que emite ondas electromagnéticas debe experimentar una pérdida de masa igual a $L / V^{2}$ (donde $L$ es la energía y $V$ la velocidad de la luz) (Einstein 1905c, p. 641). Einstein siempre interpretó esta equivalencia como una suerte de identidad, en el sentido de que "masa y energía son esencialmente lo mismo" y que, por lo tanto, "la masa de un cuerpo es una medida de su contenido energético" (Einstein 1905c, p. 641). Ahora bien, si la energía irradiada por una fuente no necesitaba un soporte material cuando se encontraba como cuerpo masivo, ¿por qué habría de necesitarlo cuando se encontraba como radiación libre? El argumento depende, por cierto, de la manera en que se conciba la relación entre masa y energía en la RE, pero, en principio, la interpretación de Einstein parece verosímil. En nuestra opinión, éste es el único argumento enteramente relativista que Einstein ofreció a favor de la autosubsistencia del campo electromagnético. ${ }^{11}$

En síntesis, Einstein formuló en el marco de la RE dos argumentos independientes entre sí contra la necesidad de postular la existencia del éter electromagnético. El primer argumento, basado en el principio de relatividad, prueba que es posible prescindir del éter como referencial privilegiado para la formulación de las leyes físicas. El segundo argumento, fundado en la equivalencia entre masa

${ }^{11}$ Advirtamos una diferencia importante entre las dos formas en que puede presentarse la energía. Como materia ponderable no podría moverse a la velocidad de la luz, pero como forma de "energía ondulatoria" sí. Sin embargo, en 1905a, Einstein concibió los cuantos de luz moviéndose con velocidad $c$, antes de establecer que la materia no podía adoptar esa velocidad. 
y energía, intenta mostrar que también se puede prescindir del éter como sustrato del campo electromagnético. Admitiendo la validez de ambos argumentos, puede decirse que la RE eliminó dos de las tres funciones del éter tradicional. Einstein, sin embargo, no ofreció ningún argumento que permitiera prescindir de la función del éter como vehículo de la interacción electromagnética por acción próxima, esto es, no probó que la RE fuera capaz de evitar la acción a distancia una vez que se hubo desecho del éter. Al contrario, esta teoría resultó compatible con la acción a distancia de la fuerza electromagnética, como se advirtió tiempo después.

\section{La reivindicación del éter en la relatividad general}

Einstein parece haber creído por mucho tiempo que la RE implicaba la prescindibilidad de toda función del éter en la física a tal punto que ya en 1909 afirmó: "hoy debemos considerar la hipótesis del éter como un punto de vista superado" (Einstein 1909, p. 817). La mayoría de los físicos no aceptó en ese momento este juicio categórico. Por ejemplo, después de 1905 Lorentz siguió desarrollando su electrodinámica, basada esencialmente en la hipótesis de un éter inmóvil. Diversos físicos británicos, como Oliver Lodge y Oliver Heavyside, persistieron en la búsqueda de modelos mecánicos del éter. Por su parte, los experimentos destinados a medir el viento de éter continuaron hasta la década de 1930. El fracaso reiterado de tales experimentos hizo pensar a algunos que la hipótesis del éter se encontraba en crisis, pero no los llevó a descartarla inmediatamente. ${ }^{12}$

Cuando Einstein, en 1916, publicó la versión final de la teoría de la RG, pensó que esta teoría tenía como consecuencia la eliminación de la objetividad del espacio y el tiempo. En efecto, según su opinión, la equivalencia de todos los sistemas de coordenadas para la formulación de las leyes de la física implicaba que las coordenadas

${ }^{12}$ La persistencia, después de 1905, de una electrodinámica basada en el éter ha sido objeto de discusiones y evaluaciones contrapuestas por parte de los filósofos de la ciencia. Zahar (1989) defendió enfáticamente la racionalidad del programa de Lorentz, que considera progresivo incluso después de la formulación de la RE. Janssen (2002), por su parte, sostuvo con nuevos argumentos la superioridad del programa de Einstein. Brown (2005) defendió las hipótesis de Lorentz en contra de la frecuente acusación de que eran puramente ad hoc. Lo cierto es que, de hecho, todavía a comienzos de la década de 1930 el éter no había desaparecido de la física. Así, Lorentz, Michelson, Lodge y Heavyside, entre otros, mantuvieron hasta sus últimos días la hipótesis del éter electromagnético. Sin embargo, hay quienes, como Torretti (2007), interpretan que ya en tiempos de Maxwell se habían dado las condiciones para prescindir del éter en la formulación de una teoría electromagnética. 
espacio-temporales carecían de significado físico. La covariancia general de las ecuaciones físicas tenía como consecuencia que sólo los eventos eran objetivos, mientras que los sistemas de referencia eran puramente convencionales. En su artículo fundamental lo expresó de esta manera:

Que este requisito de covariancia general, el cual quita el último resto de objetividad al espacio y el tiempo, es un requisito natural puede verse a partir de la siguiente consideración. Todas nuestras constataciones espacio-temporales conducen siempre a la determinación de coincidencias espacio-temporales. (Einstein 1916a, p. 774)

Pero pronto cambió de idea respecto de la falta de objetividad del espacio-tiempo tras un intercambio de cartas con Lorentz en junio de 1916, donde por primera vez sugirió la identificación del espaciotiempo con un éter relativista. ${ }^{13}$ Einstein no anunció su idea de un nuevo éter relativista sino hasta 1918. Ese año publicó un artículo de carácter divulgativo en el que, bajo la forma de un diálogo imaginario con un crítico de la teoría de la relatividad, responde a eventuales objeciones a su teoría. ${ }^{14}$ En un único pasaje dedicado al éter, Einstein presenta de manera casi completa su reivindicación del éter en un contexto relativista:

[el éter] ha tenido una suerte cambiante, y no puede decirse en absoluto que ahora esté muerto. [...] De acuerdo con la teoría especial de la relatividad, ya no existe más un estado de movimiento privilegiado; esto significa la negación del éter en el sentido de las teorías anteriores. Pues si hubiera un éter, éste debería tener en cada punto espacio-temporal un determinado estado de movimiento, el cual debería desempeñar un papel en la óptica. Pero tal estado de movimiento privilegiado no existe, como enseña la teoría de la relatividad especial, y por lo tanto tampoco existe un éter en el viejo sentido. La teoría de la relatividad general tampoco reconoce un estado de movimiento privilegiado en algún punto, que pudiera interpretarse de cierta manera como la velocidad de un éter. Pero mientras que, según la teoría de la relatividad especial, una parte del espacio sin materia y sin campo electromagnético aparecería como completamente vacía, esto es, no caracterizada por ninguna cantidad física, según la teoría de la relatividad general incluso un espacio

${ }^{13}$ Véase Kostro 2000 (pp. 65-74 y 198-202), que transcribe completas la carta de Lorentz y la respuesta de Einstein.

${ }^{14}$ Muy probablemente ese crítico sea Lenard, que ya había iniciado la célebre campaña antirrelatividad en Alemania (véase Kostro 2000, pp. 75-77). 
vacío en este sentido tiene cualidades físicas, las cuales están caracterizadas matemáticamente por los componentes del potencial gravitatorio, que determina el comportamiento métrico de esta parte del espacio, así como su campo gravitatorio. Se puede entender muy bien este estado de cosas hablando de un éter, cuyo estado varía continuamente de un punto a otro. Sólo hay que ser cuidadoso para no atribuir a este "éter" propiedades materiales (por ejemplo, una velocidad determinada en cada posición). (Einstein 1918d, pp. 701-702)

Después de 1918, Einstein incluso se retractó en numerosas ocasiones de su antigua opinión, según la cual la RE había eliminado el éter de la física. En un extenso trabajo finalizado a comienzos de 1920, que dejó sin publicar, abordó con detalle la cuestión del éter. Allí, después de exponer el argumento de la equivalencia de todos los referenciales inerciales, declaró:

Por esta razón, mi opinión en 1905 era que no se debía hablar más acerca del éter en la física. Pero este juicio era demasiado radical, como veremos en las consideraciones que siguen sobre la teoría de la relatividad general. Más bien, todavía es posible suponer un medio que llena el espacio cuyos estados pueden imaginarse como campos electromagnéticos (y quizá también como materia). Pero no es permisible atribuir a este medio estados de movimiento en cada punto, a semejanza de la materia ponderable. Este éter no debería imaginarse como si consistiera en partículas cuya identidad pudiera rastrearse en el tiempo. (Einstein 1920a, p. 260)

El 17 de octubre de 1920, Einstein ofreció en Leyden, ante la presencia de Lorentz, una conferencia inaugural dedicada exclusivamente a presentar su nueva concepción de un éter relativista. Allí expuso con detalle todos los argumentos que de una manera muy compacta ya había presentado públicamente en su artículo de 1918. Formuló la idea central acerca del nuevo éter — su carencia de todo estado de movimiento — en un lenguaje relativista de la siguiente manera:

Es posible concebir objetos físicos extendidos para los cuales el concepto de movimiento no puede encontrar ninguna aplicación. Éstos no deben concebirse como compuestos de partículas cuyo curso a través del tiempo pudiera seguirse individualmente. En el lenguaje de Minkowski, esto se expresa así: no toda entidad extendida en el mundo cuadridimensional puede considerarse compuesta de líneas de mundo. (Einstein 1920b, p. 10) 
En la conclusión de su conferencia señaló explícitamente que el éter gravitatorio es también una condición para la propagación de la luz, que no podría producirse en el vacío:

podemos decir que según la teoría de la relatividad general el espacio está dotado de propiedades físicas; en este sentido, entonces, existe un éter. Según la teoría de la relatividad general, el espacio sin éter es impensable, pues en tal espacio no sólo no habría propagación de la luz, sino tampoco ninguna posibilidad de existencia de reglas y relojes, y, por lo tanto, ningún intervalo espacio-temporal en el sentido de la física. (Einstein 1920b, p. 15)

En los dos escritos principales que posteriormente dedicó al éter, ${ }^{15}$ Einstein insistió en su identificación entre el éter, el campo gravitatorio y el espacio-tiempo, así como en la imposibilidad de concebir un espacio vacío de éter (Einstein, 1924 y 1934a). Señaló que así como en las teorías de la electrodinámica clásica, el campo electromagnético se concebía como un estado del éter, después de la RG los campos debían concebirse como "estados físicos del espacio" (Einstein 1934a, p. 143). El éter despojado de toda propiedad mecánica no podía distinguirse del espacio-tiempo mismo, pero ahora éste adquiría las propiedades físicas del propio éter.

Einstein siempre sostuvo que la RG impedía pensar el espaciotiempo como un vacío. En sus trabajos más tardíos señala que un espacio-tiempo vacío de éter (o sea, de campos) no sólo no tendría estructura métrica, como ya había afirmado en 1920, sino tampoco estructura topológica. En uno de sus últimos escritos se expresó sobre este punto de la siguiente forma:

Si imaginamos que se elimina el campo gravitatorio, es decir, las funciones $g_{i k}$, no quedará ningún espacio $[\ldots]$, ni siquiera un "espacio topológico", pues las funciones $g_{i k}$ describen no sólo el campo, sino también las propiedades estructurales topológicas y métricas de la variedad. (Einstein 1954, p. 375)

De la imposibilidad de concebir la existencia de un espacio-tiempo sin propiedades físicas (al que se refiere casi siempre como "espacio"), Einstein concluye que, en lo esencial, los plenistas como Descartes tenían razón al sostener que no puede existir el espacio vacío. Desde el

\footnotetext{
${ }^{15}$ En 1929 y 1930, Einstein escribió una serie de artículos y conferencias sobre este tema (véase Kostro 2000, pp. 230-231), pero las ideas principales se encuentran todas resumidas en su artículo de 1934.
} 
punto de vista relativista, la idea cartesiana puede expresarse diciendo simplemente que "no existe espacio "vacío de campo" (Einstein 1954, p. 376). Einstein llega incluso a concebir el campo no como una entidad que llena un espacio previamente existente, sino como la realidad fundamental que constituye el espacio-tiempo mismo. De esta manera, el espacio-tiempo no es una entidad sustancial que exista por sí misma, sino algo derivado del campo: el espacio-tiempo, según sus palabras, "existe sólo como una cualidad estructural del campo" (Einstein 1954, p. 375).

Señalemos que un hecho adicional que Einstein podría haber invocado para argumentar a favor del éter era la existencia de ondas gravitatorias. Después de haber formulado las ecuaciones de campo de la RG en 1916, Einstein investigó la manera en que se propagaba la fuerza de gravedad y probó que esta teoría predecía la existencia de ondas gravitatorias que debían transmitirse en el espacio-tiempo a una velocidad finita igual a la de la luz en el vacío (Einstein 1918a). Ahora bien, cabría preguntar, como para el caso de la luz, cuál sería el sustrato de tales ondas, y la respuesta más razonable sería que es el éter gravitatorio y que, por lo tanto, las ondas gravitatorias no serían sino oscilaciones de este mismo éter.

En 1920, Einstein pensó que la introducción del éter relativista constituía el último paso en el proceso de desmecanización del éter realizado por Lorentz:

Una reflexión más cuidadosa enseña que la eliminación del éter mediante la relatividad especial no es necesaria. Se puede suponer la existencia de un éter; sólo se debe renunciar a atribuirle un estado de movimiento determinado, esto es, se debe sacar de él por abstracción la última propiedad mecánica que Lorentz le había dejado [o sea, el reposo absoluto]. (Einstein 1920b, p. 9)

¿Es en realidad este éter no mecánico muy diferente del que había postulado Lorentz? Aparentemente, como lo muestra el siguiente pasaje, ya en 1895 Lorentz había comprendido que el concepto de movimiento no se aplicaba al éter electromagnético y que, por consiguiente, no podía decirse, al menos en sentido literal, que se encontraba en reposo absoluto:

Que no se puede hablar del reposo absoluto del éter se comprende de suyo; la expresión ni siquiera tendría sentido. Cuando, para abreviar, digo que el éter está en reposo, con ello sólo quiero dar a entender que una parte de este medio no se desplaza respecto de otras y que todos los 
movimientos de los cuerpos celestes son movimientos relativos respecto del éter. (Lorentz 1895, p. 4)

En este sentido, el éter de Lorentz cumplía la función exclusiva de ser el referencial en reposo absoluto a partir del cual referir formalmente el movimiento de los cuerpos y la radiación. Pero no se encontraba en un estado de reposo mecánico, tal como un cuerpo que pudiera ponerse en movimiento absoluto.

La posición a la que llega Einstein es, en síntesis, que el éter gravitatorio, el espacio-tiempo y el campo gravitatorio son idénticos. Las tres expresiones devienen sinónimos en su vocabulario a partir de 1918. ${ }^{16}$ El éter relativista que Einstein reivindica es, entonces, un éter gravitatorio, pero el éter que había declarado superfluo en 1905 era el éter electromagnético. Después de 1920, la situación era la siguiente: la RG implica la existencia de un éter gravitatorio, mientras que la RE es compatible con la existencia del éter electromagnético, es decir, no lo prohíbe. Ahora bien, ¿es posible identificar a este último con el espacio-tiempo o se trata, más bien, de dos éteres diferentes? En principio, el campo electromagnético y el gravitatorio son campos diferentes, que como es bien conocido, Einstein se empeñó infructuosamente en unificar en un único campo total. En su conferencia de 1920 formuló la diferencia entre los dos éteres diciendo que si bien que es imposible concebir una región del espacio desprovista de potenciales gravitatorios, esto es, vacía de éter gravitatorio, resulta perfectamente posible pensar, en cambio, en una región del espacio sin un campo electromagnético y, en consecuencia, vacía de éter electromagnético. Concluyó entonces que: "El campo electromagnético, en oposición al campo gravitatorio, parece en cierto sentido ligado al éter sólo secundariamente, en tanto que la naturaleza formal del

\footnotetext{
${ }^{16}$ Véase Kostro 2002, pp. 183-185. Después de 1938, Einstein deja de utilizar el término "éter" y prefiere hablar simplemente de campos o del "campo total". Su última discusión publicada acerca del éter se encuentra en el popular libro escrito en colaboración con Infeld (Einstein e Infeld 1938, especialmente las pp. 172-185). Un pasaje especialmente interesante expresa: "Todos nuestros intentos de hacer real el éter fracasaron. Éste no reveló ni su construcción mecánica ni su movimiento absoluto. [...] Nuestros intentos de descubrir las propiedades del éter condujeron a dificultades y contradicciones. Después de estas malas experiencias, éste es el momento de olvidarse completamente del éter y de tratar de no mencionar nunca más su nombre. Diremos: nuestro espacio tiene la propiedad física de transmitir ondas, y así omitimos el uso de la palabra que hemos decidido evitar. [...] La omisión de una palabra de nuestro vocabulario, por supuesto, no es ningún remedio. ¡Nuestros problemas son verdaderamente mucho más profundos para ser resueltos de esta manera!" (Einstein e Infeld 1938, pp. 184-185).
} 
campo electromagnético no está determinada en absoluto por el éter gravitatorio" (Einstein 1920b, p. 14).

Sin embargo, la RG tiene consecuencias sobre cómo debe concebirse el campo electromagnético y, por consiguiente, sobre el respectivo éter. Es interesante advertir, entonces, cómo la RG relaciona ambos campos. Según esta teoría, el campo electromagnético, si existe, contribuye a la curvatura del espacio-tiempo (dado que interviene en el tensor energía-impulso que sirve de fuente de la curvatura en la ecuación de Einstein), por lo cual, en términos de Einstein, codetermina, junto con la materia, la "estructura" del propio éter gravitatorio. En efecto, el principio de equivalencia empleado por Einstein postula que, localmente, todas las leyes físicas, en ausencia de campos puramente gravitatorios, son equivalentes a las de la RE. ${ }^{17} \mathrm{La}$ forma de aplicar este principio es formulando de manera covariante las diferentes ecuaciones físicas, entre ellas las ecuaciones de Maxwell, teniendo en cuenta que localmente, en ausencia de gravedad, vale la RE. Dado que no existe situación donde la gravedad esté completamente ausente, el espacio-tiempo vacío de Minkowski queda reducido a un caso límite del espacio-tiempo de la RG. Ahora bien, la validez de la RE se postula localmente, esto es, en una región del espaciotiempo muy aproximadamente plana (dependiendo de la intensidad del campo gravitatorio en las vecindades). Muy lejos de las fuentes, donde la estructura del espacio-tiempo tiende a ser la de Minkowski, el éter se convierte en una entidad cada vez más "innecesaria" dado que no hay interacciones. En estas regiones no habría campos, ni ondas de ningún tipo (tampoco ondas electromagnéticas) por lo que el éter sería verdaderamente superfluo. Incluso en el caso de una partícula aislada, ésta no evolucionaría en un espacio-tiempo plano, ya que el mismo estaría curvado a causa de su propia presencia (además, una partícula con carga eléctrica no produciría la misma

\footnotetext{
${ }^{17}$ Einstein casi siempre se refiere a este principio en términos muy generales como cuando afirma que "inercia y gravedad son fenómenos de idéntica naturaleza" (Einstein 1918b, p. 241). En las formulaciones actuales de la RG es usual distinguir varias especies diferentes de principios de equivalencia, algunos de ellos más fuertes que otros. Según Clifford Will, el principio de equivalencia de Einstein incluye tres componentes diferentes: a) el principio de equivalencia débil, que afirma que la trayectoria de un cuerpo en caída libre (no sometido a otras fuerzas aparte de la gravedad) es independiente de su estructura interna y composición; el principio de invariancia local de Lorentz, según el cual el resultado de cualquier experimento no gravitatorio local es independiente de la velocidad del referencial en caída libre en el cual se lo realiza; y el principio de invariancia local de posición, según el cual el resultado de cualquier experimento no gravitatorio local es independiente del momento y el lugar del universo en el cual se lo realiza (Will 1993, pp. 22-23).
} 
curvatura que una partícula neutra). Si sólo la RG proporciona una descripción correcta de la estructura del espacio-tiempo, y la RE queda reducida a un caso límite de aquélla, entonces, el éter sólo existe en las regiones donde existen campos. Donde no hay campo, no hay materia ni radiación, y si identificamos el éter gravitatorio con el campo gravitatorio, y éste con las propiedades del campo de la métrica, un espacio-tiempo carente de toda distribución de masa y energía, carecería de propiedades geométricas. Ésta es la idea, que Einstein repitió una y otra vez, según la cual un espacio vacío de campos es inconcebible. ${ }^{18}$ Observemos, no obstante, que para el caso de la solución de Schwarzschild, cuando la masa tiende a cero, la métrica del espacio-tiempo tiende a la de Minkowski, de forma tal que en ausencia de toda masa, el espacio-tiempo de Minkowski resultante carecería, de hecho, de todo campo gravitatorio y de todas las propiedades físicas asociadas a él. A diferencia del espacio-tiempo de la RG estaría despojado de propiedades físicas. Ahora bien, el espacio-tiempo de Minkowski, si bien de por sí tiene propiedades geométricas (que son las conocidas de la RE), en el marco de la RG constituye un caso límite que no representa una situación física real, en el sentido de que un tal espacio-tiempo estaría desprovisto de campo gravitatorio y no constituiría el escenario de ninguna interacción física. ${ }^{19}$

\section{Un ejemplo de cambio conceptual}

Como ya hemos señalado, Einstein identifica el éter gravitatorio con el campo gravitatorio, y este campo con el espacio-tiempo de la RG, al que denomina campo métrico. ${ }^{20}$ Es evidente que no es esto a lo que se

${ }^{18}$ El caso de un espacio-tiempo dotado de curvatura variable en ausencia de fuentes de materia, como el proveniente de la solución de De Sitter (1917), era rechazado por Einstein cuando indicaba que no cumplía con las condiciones del principio de Mach, según el cual el campo métrico debía estar completamente determinado por la distribución de masa y energía (Einstein 1918c).

${ }^{19}$ La situación es diferente en el marco de la RE donde el espacio-tiempo de Minkowski, por principio, posee propiedades geométricas perfectamente determinadas e inalterables, incluso inconmovibles ante la presencia de cualquier masa. De todos modos, debe reconocerse que Einstein nunca explicó claramente por qué consideraba físicamente imposible que el espacio-tiempo de la RG sea globalmente minkowskiano.

${ }^{20}$ En la formulación actual, los modelos del espacio-tiempo de la RG se representan como una estructura $\langle M, g\rangle$, donde $M$ es una variedad diferenciable de cuatro dimensiones $\left(M=\mathbb{R}^{4}\right)$, y $g$ es una métrica semirriemanniana de signatura $(1,3)$, la cual se relaciona con la distribución de materia y energía, expresada en el tensor momento-energía $\boldsymbol{T}$, de acuerdo con las ecuaciones de campo de Einstein. El éter 
llamaba éter en la teoría ondulatoria de la luz o en la electrodinámica de Maxwell. El propio Einstein lo reconoce explícitamente en su conferencia de 1920, en la que afirma que en el contexto de la RG:

el concepto de éter ha adquirido nuevamente un contenido claro, aunque un contenido que es muy diferente de aquel del éter de la teoría mecánica ondulatoria de la luz. El éter de la teoría general de la relatividad es un medio que en sí mismo está desprovisto de propiedades mecánicas y cinemáticas, pero codetermina los fenómenos mecánicos (y electromagnéticos). (Einstein 1920a, p. 19)

Señalemos también que la relación conceptual entre el espaciotiempo de la RG y el éter ya había sido advertida, de manera independiente, por Hermann Weyl y Arthur Eddington. En 1918, Weyl había señalado que el campo métrico que describen los coeficientes $g_{i k}$ podía llamarse "éter" más que campo gravitatorio (Weyl 1918, p. 182). Por su parte, Eddington propuso y luego desarrolló un concepto similar de "éter relativista" (Eddington 1920, p. 187). Advirtamos que los libros de Weyl y Eddington fueron escritos antes de que Einstein pronunciara su conferencia sobre el éter en octubre de 1920.

Inmediatamente después de la conferencia de Einstein en la que éste reintroduce el éter, Max Born, en un libro publicado en el mismo año, reaccionó sugiriendo que se había producido un cambio en el concepto mismo:

Recientemente, Einstein propuso llamar al espacio equipado con campos gravitacionales y electromagnéticos el "éter", donde, sin embargo, no debe denotar una sustancia con sus atributos tradicionales. Por consiguiente, en el "éter" no debe haber puntos determinables, y no tiene sentido hablar de movimiento relativo al "éter". Tal uso de la palabra "éter" es por cierto admisible y, una vez que haya sido sancionado de esta manera por el uso, probablemente bastante conveniente. (Born 1962, p. 224)

¿Se había vuelto equívoco el término “éter”? Weyl fue más explícito al respecto y en 1922 se refirió a la reintroducción del término éter en el contexto de la RG de esta manera:

gravitatorio de la RG debe identificarse con la totalidad de la estructura $\langle M, g\rangle$, pero no con la variedad diferenciable $M$ por sí sola, ya que el éter es el campo gravitatorio mismo y, por consiguiente, está dotado de propiedades métricas. 
ahora Einstein habla nuevamente de un éter en la relatividad general. Pero la identidad de la palabra no debería confundirnos acerca de la diferencia de la cosa. [...] El éter sustancial fue concebido por sus inventores como algo real, comparable a los cuerpos ponderables. En la electrodinámica de Lorentz se transformó en una estructura puramente geométrica, es decir, fija de una vez y para siempre, no influida por la materia. En la teoría de la relatividad especial de Einstein se puso en su lugar otra estructura, la de la geometría afín. Finalmente, en la teoría general de la relatividad, esta última se transformó en la "conexión más afín" o el "campo guía", y se convirtió en un campo de estados dotado de realidad física en interacción con la materia. $\mathrm{Y}$ por eso Einstein consideró necesario reintroducir la vieja palabra éter para un concepto completamente transformado; si esto fue conveniente o no es menos una cuestión física que filológica. (Weyl 1922, p. 59)

Ese mismo año, Lorentz, en sus lecciones de física teórica, se hizo eco del mismo problema:

haya o no un éter, los campos electromagnéticos ciertamente existen, así como la energía de las oscilaciones eléctricas. Si no nos gusta el nombre de "éter", debemos usar otra palabra como una clavija para colgar todas estas cosas. No es claro si "espacio" puede extenderse de tal manera que dé cuenta no sólo de las propiedades geométricas, sino también de las eléctricas. (Lorentz 1922, p. 210)

Indudablemente se había producido un cambio conceptual y el significado del término ya no era el mismo. No era, sin embargo, la primera vez que el concepto de éter cambiaba de significado. En un pasaje de su libro con Infeld, Einstein reconocería este hecho con las siguientes palabras:

Todavía podemos usar la palabra éter, pero sólo para expresar las propiedades físicas del espacio. La palabra éter ha cambiado muchas veces su significado en el desarrollo de la ciencia. Actualmente no designa un medio hecho de partículas materiales. Su historia no está terminada de ningún modo, sino que es continuada por la teoría de la relatividad. (Einstein e Infeld 1938, pp. 159-160)

De hecho, la comunidad de los físicos, salvo excepciones momentáneas, como las representadas por Weyl y Eddington, no siguió a Einstein en su introducción de un éter relativista. El concepto mismo de éter cayó en desuso durante la década de 1930 y el propio Einstein lo abandonó después de 1938. Los físicos prefirieron emplear el 
concepto de campo para designar aquello que Einstein había llamado "éter" y ese uso se ha mantenido hasta la actualidad.

En nuestra opinión, el problema terminológico es mucho menos trivial de lo que Born y Weyl habían pensado. Ante todo, las cuestiones lingüísticas a veces tienen una enorme importancia teórica y pueden influir decisivamente en el curso de la investigación. Por otra parte, aquí no se trata solamente de una cuestión de uso lingüístico, sino de un problema conceptual, en el sentido de que el término éter no es un nombre propio puramente referencial que, por carecer de intensión, pudiera elegirse de manera arbitraria o puramente convencional. Es un nombre de clase que tiene un significado intensional $\mathrm{y}$, por consiguiente, involucra un concepto. Por otra parte, es un predicado que ya había sido utilizado ampliamente en las teorías físicas prerrelativistas con un significado ciertamente ambiguo, pero relativamente exento de vaguedad. El problema consiste, entonces, en determinar qué relación tiene el nuevo significado asignado por Einstein al término éter con el antiguo significado (o significados) que ese término tenía en la electrodinámica clásica. ¿Estamos tal vez ante un caso de inconmensurabilidad léxica, como los que describió Kuhn? ${ }^{21}$ Nuestra posición es que los diferentes significados de "éter" son comparables y que la comparación entre ellos muestra que hay una continuidad importante en la connotación del éter electromagnético y en la del éter relativista.

Es indudable que durante el siglo XIX no sólo cambió el significado del término "éter", sino que durante largo tiempo coexistieron varios sentidos diferentes e incompatibles del mismo. El cambio de significado, evidentemente, no se produjo de manera lineal y uniforme. Cada significado parcialmente diferente de "éter" no fue una modificación de otro que lo precedió inmediatamente. Durante el último tercio del siglo, existieron varias líneas de desarrollo del concepto de éter, pero parece evidente que una de ellas condujo directamente desde el éter material con propiedades mecánicas especiales de Maxwell hasta el éter completamente desmecanizado de Einstein con la intermediación del éter en reposo absoluto de Lorentz. ${ }^{22}$

${ }^{21}$ Por ejemplo, en Kuhn 1974 y 1983. Para un estado de la cuestión, véase Arabatzis y Kindi 2008.

${ }^{22}$ Un esquema de este proceso es el siguiente. Para Maxwell, el éter electromagnético era una sustancia material semejante a la materia ponderable ordinaria, pero "de un tipo más sutil" (Maxwell 1875, p. 763). Para Lorentz, en cambio, el éter era un referencial privilegiado para la formulación de las leyes de la electrodinámica, cuya propiedad fundamental era la inmovilidad, mientras que las restantes propiedades mecánicas que pudiera tener no resultaban relevantes para las leyes físicas. 
Nos parece claro que el concepto de éter sufrió una serie de cambios de significado antes y después de la formulación de la RE. Pero se trata de cambios graduales, donde algunas de las propiedades del antiguo éter luminífero todavía se mantienen. Es evidente que el éter electromagnético pasó por un proceso de desmaterialización: perdió las propiedades características de los cuerpos de la física newtoniana y cada vez se volvió menos distinguible del espacio mismo. La identificación del éter gravitatorio con el espacio-tiempo que hace Einstein parece un cambio radical de la noción de éter si se la compara con el sólido elástico que postulara Huygens o con la sustancia material compuesta de moléculas en rotación que conjeturaba Maxwell. Resulta, en cambio, menos diferente del éter inmaterial de Lodge y todavía menos del éter inmóvil sin propiedades mecánicas de Lorentz. La concepción del éter de Einstein puede verse efectivamente como la culminación de un proceso gradual de desmaterialización y desmecanización del éter electromagnético. En el límite, sólo queda identificar al éter con el espacio mismo.

Michelson, Lodge y otros teóricos del éter admitieron que, si acaso podía suponerse material, el éter no era materia ordinaria (véanse los cálculos de Michelson sobre la bajísima densidad o la enorme elasticidad del éter en Michelson 1903, pp. 146-147, que muestran las propiedades extraordinarias que debían atribuirse al éter). Para Lodge, el éter era "la sustancia o sustrato material del cual la materia está compuesta", y se diferenciaba de ésta por carecer de todo movimiento (Lodge 1909, pp. 117 y 118). Lorentz intentó conciliar la idea de partículas en movimiento con la del éter estacionario suponiendo que las partículas mismas eran "modificaciones locales en el estado del éter", capaces de "viajar hacia adelante mientras que los elementos de volumen del medio en el cual existen permanecen en reposo" (Lorentz 1916, p. 11; la misma afirmación se encuentra mucho antes en sus escritos, por ejemplo, en su discurso por el Nobel de 1902). La idea de que el éter es la auténtica sustancia del universo y la materia, es decir, las partículas, una realidad derivada o una suerte de epifenómeno, constituye el fundamento de la llamada concepción electromagnética del mundo, que ya se hallaba ampliamente difundida entre los teóricos del éter a fines del siglo XIX y pervivió hasta las primeras décadas del siglo XX. Lodge, por ejemplo, la había expresado concisamente en 1889 diciendo que el éter mismo constituía la materia, la electricidad y la luz, y transmitía por continuidad toda acción y reacción (Lodge 1899, p. 358). Einstein recibió una profunda influencia de esta concepción del mundo, posiblemente a través de Lorentz, y la sostuvo después de la formulación de la RG hasta el final de su vida. Su programa de una teoría del campo unificado, al que consideraba como la culminación del enfoque de Maxwell (Einstein 1931) no difería demasiado de las ideas de Lodge, ya que consistía, esencialmente, en reducir toda la materia y todas las fuerzas de la naturaleza al éter (o lo que era equivalente, al campo unificado). Este tipo de programa de unificación lo continuó la geometrodinámica de John Wheeler durante la década de 1960, hasta que lo abandonó en la década siguiente cuando resultó claramente superado por el éxito del modelo estándar de partículas elementales (véanse Wheeler 1962 y Graves 1971). 
Es evidente que el significado del concepto de éter se modificó, pero ese cambio sólo fue parcial puesto que si bien perdió algunas de las propiedades del éter tradicional, retuvo otras que podrían considerarse fundamentales y que habrían de legitimar persistir en el uso del término. Así, podemos decir que el significado de "éter" cambió en cuanto éste deja de ser necesariamente homogéneo e isótropo, ya no constituye un referencial privilegiado necesario para interpretar las leyes de Maxwell, y queda desprovisto de cualquier propiedad mecánica, incluso el movimiento o el reposo. Es obvio que se trata de algo muy diferente de un sólido elástico o un fluido sutil como el que trataban de describir los modelos mecánicos del éter sólo unas décadas antes de Einstein.

A su vez, podemos considerar que el significado de "éter" no cambió en la medida en que el espacio-tiempo dotado de propiedades físicas al que Einstein llama el "éter", aunque no sea una entidad material, conserva muchas de las propiedades del éter luminífero tradicional. Ante todo, es una entidad continua, la única en la naturaleza. Constituye, asimismo, una condición necesaria para la propagación de la luz y también para la existencia de campos y ondas, por ejemplo, para las ondas gravitatorias. Por último, la existencia de este éter es también necesaria para evitar la acción a distancia en las interacciones electromagnética y gravitatoria. Dos de las tres funciones del éter tradicional aún se conservan en el éter relativista. ${ }^{23}$

La teoría de la RG representa indudablemente un cambio conceptual muy profundo en la manera de comprender los fenómenos gravitatorios respecto de la teoría de la gravedad de Newton. Para los newtonianos, la gravedad se concebía como una fuerza siempre atractiva entre cuerpos, formalmente actuando a distancia, y que sólo dependía de la masa y de la posición de dichos cuerpos. Para Einstein, en cambio, la gravedad no es sino una manifestación de la estructura métrica del espacio-tiempo que depende de la distribución de materia y energía. De forma tal que es suficiente conocer la estructura y la dinámica del espacio-tiempo (y las condiciones iniciales y de contorno) para determinar el movimiento de los cuerpos. La gravedad, además, no sólo afecta el movimiento de las partículas y

${ }^{23}$ Al final de su segundo artículo sobre el éter, Einstein señala la necesidad del éter para evitar la acción a distancia en estos términos: "no podremos prescindir del éter en la física teórica, esto es, de un continuo dotado de propiedades físicas, pues, la teoría de la relatividad general, cuyos puntos de vista básicos los físicos seguramente mantendrán siempre, excluye una acción inmediata a distancia; pero toda teoría de acción próxima presupone campos continuos, y por lo tanto también la existencia de un "éter" " (Einstein 1924, p. 93). 
cuerpos materiales, sino también la propagación de la luz, la que siempre debe moverse a lo largo de curvas geodésicas nulas compatibles con la métrica espacio-temporal. Como consecuencia de ello, no sólo la caída de una piedra o el movimiento orbital de un satélite resultan fenómenos gravitatorios, sino también, por ejemplo, el corrimiento hacia el rojo en el espectro de la luz de una estrella muy masiva. Que el llamado corrimiento gravitatorio hacia el rojo sea un fenómeno de tipo gravitatorio representa una recategorización muy radical de los hechos. ${ }^{24}$ Esto implica que la propagación del campo electromagnético se ve afectada por la estructura del espacio-tiempo; en términos de Einstein, por el propio éter gravitatorio.

Ahora bien, ¿de qué manera se puede justificar la identificación del éter con el espacio-tiempo? La cuestión de la aplicación de un concepto físico nunca es puramente arbitraria, pero siempre se decide, como ocurre con cualquier otro concepto del lenguaje natural, por medio de los usos y las costumbres de la comunidad. Los físicos optaron por desterrar el término "éter" y adoptar el de "campo", pero, como ya hemos señalado, Einstein observó con agudeza que la simple eliminación de un término no resuelve los problemas conceptuales. De hecho, el concepto de campo en la RG (así como en las teorías de campos cuánticos) también cambió de significado y, sin duda, el campo reinstaló algunas de las propiedades características del éter. Cuando se asignan propiedades físicas a cada punto del espaciotiempo, como ocurre en toda teoría de campos, el campo mismo adquiere las propiedades de un éter desmaterializado que difícilmente puede distinguirse del éter relativista que Einstein introdujo. De manera muy acertada, Einstein resumía esta situación diciendo que “en vez de hablar de un 'éter' se podría hablar igualmente bien de "cualidades físicas del espacio"” (Einstein 1924, p. 85).

Llamar "éter" al espacio-tiempo de la RG implica, sin duda, un cambio en el significado del término, y su empleo involucra una cuestión lingüística y conceptual importante. No obstante, llamar "espacio" (para referirse al espacio físico) a este espacio-tiempo, como hizo Einstein, no plantea un problema menor. Los conceptos de espacio y tiempo se modificaron en la RE respecto de la mecánica newtoniana, y luego volvieron a cambiar en el marco de la RG.

${ }^{24}$ Torretti (2003, pp. 198-201) ha señalado este punto como un ejemplo del problema general que llama el de "la idoneidad de los conceptos". Es evidente que la clasificación de ciertos fenómenos físicos como fenómenos gravitatorios en la RG difiere de la que es resultado de la teoría newtoniana de la gravedad, pero no es evidente que sea preferible a ésta en algún aspecto. 
La diferencia entre las teorías del éter de Einstein y Lorentz se puede explicar, precisamente, tal como lo ha señalado Janssen (2002), como un cambio en la concepción del escenario mismo en el que se desarrollan los fenómenos físicos. La teoría de Lorentz era una combinación de mecánica newtoniana, que proveía el escenario (esto es, el espacio euclidiano, el tiempo absoluto y las leyes de movimiento clásicas), y la electrodinámica maxwelliana, que proporcionaba las leyes de los campos electromagnéticos concebidos como estados del éter, el cual definía un referencial absoluto para las leyes de la física. Así, el espacio y el tiempo se regían por las transformaciones de Galileo, mientras que los campos, que no eran invariantes galileanos, se regían por las transformaciones de Lorentz. En cambio, Einstein en 1905 elaboró una teoría donde se conmueve la estructura misma del espacio-tiempo: su transformación desde un sistema inercial a otro está expresada por las transformaciones de Lorentz y ninguno es privilegiado para la formulación de las leyes físicas.

En la RE, el carácter de la transformación de Lorentz es conceptualmente diferente del que tenía en la electrodinámica de Lorentz y mucho más fundamental, ya que no se refiere a determinadas leyes físicas sino al propio escenario en que ocurren todos los fenómenos. Como las leyes de la física se expresan siempre espaciotemporalmente, todas las leyes, mecánicas, electromagnéticas o de cualquier otro tipo, deben resultar, por el principio de relatividad, invariantes lorentzianos, porque lo que en el fondo se está transformando es el espacio-tiempo mismo. De esta manera, por ejemplo, la propia contracción de Lorentz se interpreta de un modo diferente: no como causada por fuerzas intermoleculares que dependen de la velocidad absoluta del objeto respecto del éter, sino como producto de la relatividad de la simultaneidad entre eventos distantes medidos en referenciales en movimiento relativo.

Ahora bien, en la RG, las nociones de espacio y tiempo sufren un nuevo cambio conceptual. Desde el punto de vista geométrico, son permisibles las transformaciones generales de coordenadas (lo que incluye referenciales acelerados). Pero además, desde el punto de vista físico, el espacio-tiempo posee un carácter dinámico ya que es deformado por la presencia de la radiación y la materia, mientras que las propias fuentes, en su evolución, "padecen" la curvatura del espacio-tiempo. Así, el espacio-tiempo de la RG constituye, de hecho, una entidad física que interactúa con la materia y la radiación, mientras que el espacio tiempo de Minkowski era tan inmutable como el espacio absoluto newtoniano en cuanto que no resultaba afectado por la presencia de materia. En la RG, que, a diferencia de la RE, 
es esencialmente una teoría de campos, el espacio-tiempo constituye un campo métrico cuyas propiedades geométricas se manifiestan en efectos físicos. En este escenario era posible, e incluso razonable, reintroducir el término éter, con un nuevo significado, para designar al campo métrico. Pero, dado que el campo métrico era un campo físico, también quedaba abierta la posibilidad de prescindir del concepto de éter y retener solamente el de campo. El propio Einstein adoptó esta postura y, como ya vimos, renunció al uso del concepto de éter relativista que él mismo había introducido. Puede decirse incluso que el concepto de éter resultó absorbido por el de campo. Pero entonces la noción de campo también se modificó y ahora el campo gravitatorio asumió algunas de las propiedades y funciones del éter mismo.

\section{6. ¿Agujeros en el éter?}

La identificación del éter relativista con el espacio-tiempo de la RG plantea inmediatamente la pregunta de si este hecho reivindica una concepción sustancialista del espacio-tiempo. De acuerdo con esta idea, los fenómenos físicos ocurren en un espacio-tiempo preexistente que es independiente de ellos. Ésta es sólo una de las nociones tradicionales de sustancia, según la cual sustancia es aquello que existe por sí mismo y no depende de otra cosa para existir, pero es la que está en juego en la discusión entre los partidarios de las concepciones relacional y absoluta del espacio y el tiempo. ${ }^{25} \mathrm{El}$ éter gravitatorio de la RG es indudablemente una entidad autosubsistente y, en tal sentido, una sustancia, aunque no de carácter material, como ya señalamos. Por onsiguiente, el espacio-tiempo de la RG tendría un carácter sustancial.

Por otra parte, ¿es el éter relativista entendido como sustancia igualmente vulnerable al conocido argumento del agujero que Earman y Norton (1987) idearon, inspirados en Einstein, contra el sustancialismo espacio-temporal ${ }^{26}$ Dicho argumento intenta mostrar

${ }^{25}$ La obra de Earman (1989) sigue siendo la referencia fundamental sobre este tema.

${ }^{26}$ Este argumento no sólo se aplica al espacio-tiempo de la RG, sino a una clase muy amplia de teorías locales del espacio-tiempo. Aquí seguiremos, de manera muy resumida, la presentación original de Earman y Norton (1987) referida a la RG, y la de Norton (1988) que extiende el argumento a otras teorías del espacio-tiempo. Earman 1989, cap. 9, contiene una discusión más extensa y detallada de todo el tema. En los últimos veinte años se han publicado un gran número de trabajos a favor y en contra del argumento del agujero, pero dar cuenta de estos debates no es nuestro objetivo en este artículo. 
que si el espacio-tiempo de la RG se concibe como una sustancia, como consecuencia se produce una forma indeseable de indeterminismo. Einstein inventó este argumento en 1913 con el fin de mostrar que las ecuaciones generalmente covariantes del campo gravitatorio no son aceptables porque violan el determinismo causal. ${ }^{27}$ Mediante una suerte de experimento mental concibió un universo lleno de materia donde existe una región finita, el agujero $A$, en la cual no hay materia ni energía (es decir, el tensor momento-energía $\boldsymbol{T}=0$ ). Se preguntó, entonces, si la distribución espacio-temporal de la materia y la energía determinaba la estructura métrica dentro del agujero, y concluyó que las curvas geodésicas quedaban indeterminadas ya que, en el interior del agujero, la métrica no era única. Después de la formulación de las ecuaciones de campo de la RG, en noviembre de 1915, Einstein no volvió a referirse a este argumento, que podría haber constituido una objeción posible para su concepción del éter gravitatorio.

En la versión de Earman y Norton (1987), el argumento del agujero toma como punto de partida la existencia de modelos difeomórficos del espacio-tiempo de la RG que son observacionalmente indistinguibles. Así, si $\langle M, g\rangle$ es un modelo de la RG que representa una situación físicamente posible, y $h$ es un difeomorfismo sobre $M$ que transforma la métrica $g$ en la métrica $h(g)=g^{\prime}$, la covariancia general de la RG garantiza que $\left\langle M, g^{\prime}\right\rangle$ también es un modelo de la RG. En general $g$ y $g^{\prime}$ serán diferentes, ya que cambian el valor de la métrica en cada evento de $M$. Sin embargo, ambos modelos son observacionalmente indistinguibles porque coinciden en los valores que atribuyen a todos los observables de la RG (tiempo propio, longitud propia, masa en reposo, etc.). ${ }^{28}$

El argumento del agujero se obtiene construyendo dos de estos modelos difeomórficos de la siguiente manera: $h$ es una transformación de identidad en $M$ excepto en cierto entorno de $M$, el agujero $A$, que puede ser tan pequeño como se quiera. De este modo, $g^{\prime}=h(g)=g$ fuera de $A$, pero $g^{\prime}=h(g) \neq g$ dentro de $A$. Este hecho implica una

${ }^{27}$ La primera formulación del argumento apareció en Einstein y Grossman 1913, pp. 260-26l. Sin duda, la finalidad del argumento era justificar el carácter no generalmente covariante de las ecuaciones del campo gravitatorio que formulaban en ese trabajo. La última formulación del argumento se encuentra en Einstein 1914, pp. 1066-1067.

${ }^{28}$ En la bibliografía sobre la RG se reconoce la existencia de diferentes modelos difeomórficos, pero se los considera equivalentes, en el sentido de que son descripciones matemáticas diferentes de la misma situación física. La transformación que lleva de uno a otro se concibe, entonces, como una simple transformación de gauge. 
extraña forma de indeterminismo. Dado que las geodésicas espaciotemporales definidas por ambos modelos sólo difieren en el interior de $A$, la teoría resulta incapaz de predecir la trayectoria de las partículas en caída libre que atraviesen el agujero, aun cuando se tenga una especificación completa de la estructura métrica de $M$ fuera del agujero.

Ahora bien, el argumento de Earman y Norton se basa en el supuesto de que el espacio-tiempo está representado exclusivamente por la variedad diferenciable $M .^{29}$ Pero éste es un supuesto que podría cuestionarse, sosteniendo que el espacio-tiempo requiere estructuras adicionales, además de $M$, ante todo una estructura métrica, ya que los eventos de una variedad "desnuda" no tienen identidad física independiente de la métrica (Hoefer 1996). Esto es evidente en el caso del éter gravitatorio, que es el campo métrico mismo. No obstante, Norton (1988) mostró que el argumento se puede extender a estructuras más ricas, que incluyan la métrica, siempre que ésta admita algunas simetrías comunes, como la homogeneidad y la isotropía. La RG es una teoría que admite estas simetrías (por ejemplo en las métricas de Friedmann-Robertson-Walker), por lo que el argumento del agujero se le aplicaría incluso suponiendo que el espacio-tiempo debe representarse mediante el par $\langle M, g\rangle$ (como sostuvimos que ocurría con el éter relativista). La posibilidad de que exista esta clase de "agujeros en el éter", por lo menos formalmente, no puede excluirse en el marco de la RG. Pero aun si se admite la corrección del argumento del agujero, éste sólo representa una objeción seria al sustancialismo si se desea mantener el carácter estrictamente determinista de la RG, como ciertamente quería Einstein. Pero dado que sustancialismo y determinismo son hipótesis independientes, en este sentido el argumento del agujero no refuta el sustancialismo espaciotemporal ni, por consiguiente, la concepción del éter relativista como una sustancia. No obstante, muestra que la posición sustancialista tiene costos metafísicos y epistemológicos, como el indeterminismo y la posible existencia de situaciones físicas observacionalmente indistinguibles.

\section{Conclusiones}

El éter relativista de Einstein fue el resultado de un proceso de cambio conceptual que, en nuestra opinión, es bastante frecuente en la

${ }^{29}$ Earman y Norton llamaron a la correspondiente variedad de sustancialismo manifold substantivalism (véase Earman 1989, pp. 125-126, para una discusión de esta expresión). 
historia de la ciencia. El surgimiento y la desaparición de este concepto ejemplifica algunos patrones típicos, aunque no necesariamente universales, de la manera en que se produce el cambio de significado en los conceptos de las teorías físicas. En primer lugar, el concepto de éter se volvió inestable, debido a razones tanto teóricas como experimentales, antes de la formulación de la RE. En segundo, aparecieron varios conceptos diferentes, pero parcialmente coincidentes, de éter. En tercer lugar, se produjo un proceso de eliminación de propiedades mecánicas del éter electromagnético, que, con Lorentz, quedó reducido a un referencial privilegiado e inobservable en estado de reposo absoluto. En cuarto lugar, con las dos teorías de la relatividad de Einstein hubo un cambio de significado conjunto de varios conceptos físicos fundamentales, como los de espacio, tiempo y campo. En este nuevo contexto resultó posible hacer la identificación entre el éter y el espacio-tiempo de la RG de una manera novedosa. Finalmente, el concepto de éter terminó siendo absorbido por el de campo, que, a su vez, también cambió de significado y adquirió algunas de las notas características del antiguo éter electromagnético.

Desde un punto de vista histórico, el éter surgió como un concepto explicativo: no describía ningún fenómeno físico observable, pero permitía explicar determinados fenómenos como la propagación de la luz en el vacío. Cuando Einstein propuso eliminar el éter en la RE, dejó sin explicar cómo se propagaba la luz en el vacío y cómo se podía evitar la acción a distancia de la fuerza electromagnética. El éter gravitatorio de la RG recuperó estas funciones explicativas, dando cuenta del sustrato de las ondas gravitatorias y de la propagación de la fuerza gravitatoria mediante acción próxima. El costo que debió pagarse por este poder explicativo fue que hubo que despojar al antiguo éter de toda cualidad mecánica. Para un físico de inclinación positivista, este éter sería una entidad ociosa que, en principio, debería eliminarse de cualquier teoría física. El propio Einstein adoptó esta actitud antes de la formulación de la RG. Dado que el éter relativista no posee ninguna propiedad que pueda ser medida directamente, su presencia y sus cualidades sólo pueden ser inferidas a partir de sus efectos en el comportamiento de la materia y la radiación; por ejemplo, en las características de las geodésicas nulas que describiría la luz en cada caso, esto es, respecto de la correspondiente solución de las ecuaciones de Einstein para el campo de la métrica.

Durante los procesos de cambio teórico podemos observar una suerte de inestabilidad de los conceptos fundamentales de las teorías vigentes. Este fenómeno se produce cuando un concepto empieza a tornarse ambiguo, cuando se pierde el consenso acerca de sus notas 
fundamentales y cuando comienza a haber dudas acerca de si es un concepto genuinamente referencial. El concepto de éter ya se había vuelto inestable en la electrodinámica de fines del siglo XIX, a partir del momento en que se trató de reconciliar ésta con la mecánica newtoniana y con los resultados negativos de los experimentos que se proponían detectar el movimiento de la Tierra respecto del éter. La materialidad del éter y su propio modo de existencia ya se hallaban cuestionados antes de la formulación de la RE y continuaron en estado problemático bastante tiempo después. Desde una perspectiva histórica, la introducción del concepto de éter relativista debe considerarse como una etapa de este proceso de cambio. Si se lo separa de dicho proceso y se lo compara con algún concepto de éter característico de las primeras etapas del cambio, por ejemplo, el éter material de Maxwell, puede producir la impresión de un cambio de significado casi completo. Pero el carácter parcial del cambio involucrado se vuelve mucho más evidente cuando se presta atención al complicado proceso de cambio conceptual, en modo alguno lineal o progresivo, que experimentó el concepto de éter en el medio siglo que separa a Maxwell de Einstein.

Los cambios teóricos más o menos considerables, como el surgimiento de la relatividad, generalmente no producen cambios conceptuales localizados, por ejemplo, en un determinado concepto, sino cambios en toda una red de conceptos relacionados. La teoría de la RE provocó cambios, entre otros, en los conceptos de espacio, tiempo, masa, energía y momento. Con la RG también cambiaron parcialmente de significado los conceptos de inercia, gravedad y campo. Si estos viejos conceptos se siguieron empleando en las nuevas teorías relativistas con significados parcialmente diferentes de los que tenían, ¿por qué la situación debía haber sido diferente con la noción de éter? La supresión del término éter del vocabulario de la física no eliminó los problemas conceptuales que la hipótesis de su existencia resolvía. Aun hoy es posible reformular estos problemas en el marco de una teoría de campos.

\section{BIBLIOGRAFÍA}

Arabatzis, T. y V. Kindi, 2008, "The Problem of Conceptual Change in the Philosophy and History of Science", en Vosniadou 2008, pp. 345-373.

Born, M., 1962, Einstein's Theory of Relativity, Dover, Nueva York. (la. ed. en alemán 1920.)

Brown, H.R., 2005, Physical Relativity: Space-Time Structure from a Dynamical Perspective, Clarendon Press, Oxford. 
Cantor, G.N. y J.S. Hodge, (comps.), 1981, Conceptions of Ether: Studies in the History of Ether Theories, 1740-1900, Cambridge University Press, Cambridge.

Cassini, A. y M.L. Levinas, 2008, "La explicación de Einstein del efecto fotoeléctrico. Un análisis histórico-epistemológico", Revista Latinoamericana de Filosofía, vol. 34, pp. 5-38.

— , 2007, "La hipótesis del cuanto de luz y la relatividad especial. ¿Por qué Einstein no las relacionó en 1905?”, Scientiae Studia, vol. 5, pp. 425-452.

- 2005, "La reinterpretación radical del experimento de MichelsonMorley en la relatividad especial", Scientiae Studia, vol. 3, pp. 547-581.

De Sitter, W., 1917, “On Einstein's Theory of Gravitation and its Cosmological Consequences", Monthly Notices of the Royal Astronomical Society, vol. 78, pp. 3-28.

Earman, J., 1989, World Enough and Space-Time: Absolute versus Relational Theories of Space and Time, The MIT Press, Cambridge, Mass.

Earman, J. y J. Norton, 1987, "What Price Spacetime Substantivalism? The Hole Story", British Journal for the Philosophy of Science, vol. 38, pp. 515-525.

Eddington, A.S., 1920, Space, Time, and Gravitation, Cambridge University Press, Cambridge.

Einstein, A., 2002, The Collected Papers of Albert Einstein, vol. 7, Princeton University Press, Princeton.

— , 1954, "Relativity and the Problem of Space", Ideas and Opinions, Crown, Nueva York, 1982, pp. 360-377.

, 1949, "Autobiographisches", en Schilpp 1949, pp. 1-95.

— 1948, “Carta a Besso del 06/01/1948”, en Einstein y Besso 1972.

Einstein 1934b, pp. 138-147.

——, 1934b, Mein Weltbild, Ullstein, Fráncfort, 1980.

__, 1931, "Maxwell Einfluss auf die Entwicklung der Auffassung des physikalisch-realen", en Einstein 1934b, pp. 159-162.

——, 1924, "Über den Äther", Vierteljahresschrift der schweizerischen naturforschenden Gesellschaft, vol. 105, pp. 85-93.

— , 1920a, "Grundgedanken und Methoden der Relativitätstheorie, in ihrer Entwicklung dargestellt", en Einstein 2002, pp. 245-278.

—_, 1920b, Äther und Relativitätstheorie, Julius Springer, Berlín.

_- 1918a, "Über Gravitationswellen", Königlich Preussische Akademie der Wissenschaften, Sitzungberichte, pp. 154-167.

— , 1918b, "Prinzipielles zur allgemeinen Relativitätstheorie", Annalen der Physik, vol. 55, pp. 241-244.

—_, 1918c, "Kritisches zu einer von Hrn. De Sitter gegebenen Lösung der Gravitationsgleichungen", Königlich Preussische Akademie der Wissenschaften, Sitzungberichte, pp. 270-272. 
Einstein, A., 1918d, "Dialog über Einwände gegen die Relativitätstheorie", Die Naturwissenschaften, vol. 6, pp. 697-702.

—_, 1916a, "Die Grundlage der allgemeinen Relativitätstheorie", Annalen der Physik, vol. 49, pp. 769-822.

_ _ 1916b, "Carta a Lorentz del 17/6/1916", en Kostro 2000, pp. 201202. 104.

, 1916c, "Ernst Mach", Physikalische Zeitschrift, vol. 17, pp. 101104.

- 1914, "Die formale Grundlage der allgemeinen Relativitätstheorie", Sitzungsberichte der Königlich Preussischen Akademie der Wissenschaften (Berlín), pp. 1030-1085.

- 1909, "Entwicklung unserer Anschauungen über das Wesen und die Konstitution der Strahlung", Physikalische Zeitschrift, vol. 10, pp. 817825.

, 1907, "Über das Relativitätsprinzip und die aus demselben gezogenen Folgerungen", Jahrbuch der Radioaktivität und Elektronik, vol. 4, pp. 411-462.

— 1905a, "Über einen die Erzeugung und Verwandlung des Lichtes betreffenden heuristischen Gesichtspunkt", Annalen der Physik, vol. 17, pp. 132-148.

— 1905b, "Zur Elektrodinamik bewegter Körper”, Annalen der Physik, vol. 17, pp. 891-921.

—, $1905 \mathrm{c}$, "Ist die Trägheit eines Körpers von seinem Energieinhalt abhängig?", Annalen der Physik, vol. 18, pp. 639-664.

_ 1901, "Folgerungen aus den Capillaritätserscheinungen", Annalen der Physik, vol. 4, pp. 513-523.

Einstein, A. y M. Besso, 1972, Correspondence 1903-1955, ed. P. Speziali. Hermann, París.

Einstein, A. y M. Grossman, 1913, "Entwurf einer verallgemeinerten Relativitätstheorie und eine Theorie der Gravitation", Zeitschrift für Mathematik und Physik, vol. 62, pp. 225-261.

Einstein, A. y L. Infeld, 1938, The Evolution of Physics, Cambridge University Press, Cambridge.

Faraday, M., 1846, "Thoughts on Ray-Vibrations", Philosophical Magazine, vol. 28, pp. 345-350. (Reimpreso en Experimental Researches in Electricity, Taylor and Francis, Londres, 1849, vol. III, pp. 447-452.)

Graves, J.C., 1971, The Foundations of Contemporary Relativity Theory, The MIT Press, Cambridge, Mass.

Hertz, H., 1890, "Über die Grundgleichungen der Elektrodynamik für bewegte Körper", Annalen der Physik, vol. 41, pp. 369-399.

Hoefer, C., 1996, "The Metaphysiscs of Space-Time Substantivalism", The Journal of Philosophy, vol. 93, pp. 5-27.

Holton, G., 1968, "Mach, Einstein, and the Search for Reality", Daedalus, vol. 97, pp. 636-673. 
Janssen, M., 2002, "Reconsidering a Scientific Revolution: The Case of Einstein versus Lorentz", Physics in Perspective, vol. 4, pp. 421-446.

Kostro, L., 2000, Einstein and the Ether, Apeiron, Montreal.

Kuhn, T.S., 1983, "Commensurability, Comparability, Communicability", reimpreso en The Road since Structure: Philosophical Essays 1970 1993, with an Autobiographical Interview, The University of Chicago Press, Chicago/Londres, 2000, pp. 33-57.

, 1974, "Second Thoughts on Paradigms", reimpreso en The Essential Tension: Selected Studies in Scientific Tradition and Change, The University of Chicago Press, Chicago/Londres, 1977, pp. 293-319.

Larmor, J., 1900, Aether and Matter, Cambridge University Press, Cambridge.

Lodge, O., 1909, The Ether of Space, Harper and Brothers, Nueva York Londres. York.

Lorentz, H.A., 1922, Lectures on Theoretical Physics, vol. 3, Macmillan, Londres.

- 1916, The Theory of Electrons and its Applications to the Phenomena of Light and Radiant Heat, 2a. Ed., Teubner, Leipzig.

- 1902, "The Theory of Electrons and the Propagation of Light", en Nobel Lectures: Physics 1901-1921, Elsevier, Amsterdam, 1967.

- - 1895, Versuch einer theorie der Elektrischen und Optischen Erscheinungen in bewegten Körpern, Brill, Leiden.

Maxwell, J.C., 1875, "Ether", Encyclopaedia Britannica, 9a. ed., vol. VIII, pp. 568-572. (Reimpreso en The Scientific Papers of James Clerk Maxwell, ed. W.D. Niven, Dover, Nueva York, 1965, vol. 2, pp. 763775.)

Michelson, M., 1903, Light Waves and their Uses, The University of Chicago Press, Chicago.

Michelson, M. y E. Morley, 1887, "On the Relative Motion of the Earth and the Luminiferous Ether", American Journal of Science, vol. 34, pp. 333345.

Norton, J., 1988, "The Hole Argument", PSA 1988, vol. 2, pp. 56-64.

Ostwald, W., 1893, Lehrbuch der allgemeinen Chemie, Engelmann, Leipzig, 2 vols.

Sánchez Ron, J.M., 1983, El origen y desarrollo de la relatividad, Alianza, Madrid.

Schaffner, K.F., 1972, Nineteenth Century Aether Theories, Pergamon Press, Oxford.

Schilpp, P.A. (comp.), 1949, Albert Einstein: Philosopher-Scientist, Open Court, La Salle.

Swenson, L.S., 1972, The Ethereal Aether: A History of the MichelsonMorley-Miller Aether-Drift Experiments, 1880-1930, University of Texas Press, Austin. 
Torretti, R., 2007, "Getting Rid of the Ether: Could Physics Have Achieved it Sooner with Better Assistance from Philosophy?", Theoria, vol. 60, pp. 353-374.

——, 2003, Relatividad y espaciotiempo, RIL Editores, Santiago de Chile.

Vosniadou, S. (comp.), 2008, International Handbook of Research on Conceptual Change, Routledge, Nueva York/Londres.

Weyl, H., 1922, "Die Relativitätstheorie auf der Naturforscherversammlung in Bad Nauheim", Jahrbericht der Deutschen Mathematikerverenigung, vol. 31, pp. 51-63.

$\longrightarrow$, 1918, Raum, Zeit, Materie, Springer, Berlín.

Will, C.M., 1993, Theory and Experiment in Gravitational Physics, ed., rev., Cambridge University Press, Cambridge.

Wheeler, J., 1962, Geometrodynamics, Academic Press, Nueva York.

Whittaker, E.T., 1953, A History of Theories of Aether and Electricity. II: The Modern Theories, 1900-1926, Nelson and Sons, Londres.

—, 1951, A History of Theories of Aether and Electricity. I: The Classical Theories, Nelson and Sons, Londres.

Zahar, E., 1989, Einstein's Revolution: A Study in Heuristics, Open Court, La Salle.

Recibido el 24 de febrero de 2009; revisado el 11 de septiembre de 2009; aceptado el 21 de octubre de 2009. 